\title{
Article
}

\section{An ANN-Based Adaptive Predistorter for LED Nonlinearity in Indoor Visible Light Communications}

\author{
Jenn-Kaie Lain * (1) and Yan-He Chen \\ Department of Electronic Engineering, National Yunlin University of Science and Technology, \\ Douliu 64002, Taiwan; qw0759@gmail.com \\ * Correspondence: lainjk@yuntech.edu.tw; Tel.: +886-5-5342601 (ext. 4335)
}

check for updates

Citation: Lain, J.-K.; Chen, Y.-H. An ANN-Based Adaptive Predistorter for LED Nonlinearity in Indoor Visible Light Communications. Electronics 2021, 10, 948. https://doi.org/ 10.3390/electronics10080948

Academic Editors: Jose A. Rabadan, Victor Guerra and Julio Rufo

Received: 20 March 2021

Accepted: 14 April 2021

Published: 16 April 2021

Publisher's Note: MDPI stays neutral with regard to jurisdictional claims in published maps and institutional affiliations.

Copyright: (C) 2021 by the authors Licensee MDPI, Basel, Switzerland. This article is an open access article distributed under the terms and conditions of the Creative Commons Attribution (CC BY) license (https:// creativecommons.org/licenses/by/ $4.0 /)$.

\begin{abstract}
By modulating the optical power of the light-emitting diode (LED) in accordance with the electrical source and using a photodetector to convert the corresponding optical variation back into electrical signals, visible light communication (VLC) has been developed to achieve lighting and communications simultaneously, and is now considered one of the promising technologies for handling the continuing increases in data demands, especially indoors, for next generation wireless broadband systems. During the process of electrical-to-optical conversion using LEDs in VLC, however, signal distortion occurs due to LED nonlinearity, resulting in VLC system performance degradation. Artificial neural networks (ANNs) are thought to be capable of achieving universal function approximation, which was the motivation for introducing ANN predistortion to compensate for LED nonlinearity in this paper. Without using additional training sequences, the related parameters in the proposed ANN predistorter can be adaptively updated, using a feedback replica of the original electrical source, to track the LED time-variant characteristics due to temperature variation and aging. Computer simulations and experimental implementation were carried out to evaluate and validate the performance of the proposed ANN predistorter against existing adaptive predistorter schemes, such as the normalized least mean square predistorter and the Chebyshev polynomial predistorter.
\end{abstract}

Keywords: artificial neural networks; digital predistorter; LED nonlinearity; visible light communications

\section{Introduction}

Light-emitting diode (LED) solid-state lighting has received considerable attention recently because of its wide applicability and advantages over conventional lighting sources for monitor backlighting, traffic indicators, and general illumination. Lighting plays a key role in people's daily lives. LEDs offer advantages such as low energy consumption, long lifetimes, small sizes, and easy production, and they are increasingly being used as replacements for incandescent lamps. LEDs are expected to become a dominant part of the next generation of lighting sources. Recently, visible light communication (VLC) has been widely studied and has become a candidate for incorporation in the IEEE 802.15.7r1 standard to achieve lighting and communications simultaneously [1]. Through intensity modulation (IM), i.e., by modulating the light-emitting power in accordance with the transmitted data, and through direct detection (DD), i.e., by detecting the subtle light intensity variation of the transmitted optical light, VLC enables the functionality of wireless communication in a manner that is relatively simple and low in cost. Notably, this communication function does not increase power and arrangement costs, and many studies have shown that VLC systems combined with Ethernet or power line communication offer a promising solution for wireless broadband access in indoor environments [2,3].

Orthogonal frequency-division multiplexing (OFDM) transmission had been considered for use in VLC systems to achieve a high data transmission rate [4]. Traditionally, the OFDM modulation and demodulation were realized by the inverse fast Fourier transform (IFFT) and the FFT, respectively. However, the traditional OFDM scheme results in 
complex-valued time-domain signals and cannot be directly applied to VLC transmission. As such, several studies have investigated optical OFDM schemes aimed at making the traditional OFDM scheme suitable for VLC systems [5-7]. Basically, the real-valued bipolar signals can be obtained by using Hermitian symmetry when allocating modulated symbols to frequency domain subcarriers, after which the IFFT can be applied. By adding a direct current bias, direct current biased optical OFDM (DCO-OFDM) converts the real-valued bipolar signals to unipolar ones $[5,6]$. On the other hand, asymmetric clipping optical OFDM (ACO-OFDM) is used to impose the anti-symmetric property and directly clip the negative parts of the real-valued bipolar signals without losing information [7]. Both DCOOFDM and ACO-OFDM have attracted considerable attention for use in VLC systems. However, LED nonlinearity severely degrades the performance of OFDM VLC systems due to the high peak-to-average power ratio (PAPR) in OFDM signals [8]. Normally, nonlinear devices are operated with a certain power backoff, which can be defined as the ratio of maximum saturation output to the average output. Increasing the power backoff reduces the nonlinear distortion, but cause lower power efficiency. Reducing power backoff is thus desirable, while the signal nonlinear distortion would seriously sacrifice bit error rate (BER) performance. Thus, LED nonlinearity not only reduces the power efficiency of such systems, but also degrades their BER performance. Although PAPR reduction schemes can decrease the PAPR in OFDM signals, both DCO-OFDM and ACO-OFDM signals still exhibit a considerable dynamic range and would be distorted by LED nonlinearity $[9,10]$.

Given this knowledge of LED behavior, the most straightforward approach to mitigating the impact of nonlinearity would be to pre-process the DCO-OFDM signal to suppress the nonlinearity in the LED emitted signal, and to possibly enable receiver side with low complexity, which is known as transmitter digital predistortion [11-13]. Initially, the LED characteristics can be learned by using training sequences. Subsequently, the adaptive predistorter is capable of tracking the LED characteristics due to temperature variation and aging by using the transmitted data sequences. The use of a lookup-table (LUT) that implements the inverse function against the nonlinearity of the LED constitutes the simplest predistorter [11]. The weight required to compensate for distortion from the nonlinearity of the LED is estimated and stored in the LUT. The transmitted signal is then predistorted by multiplying the weight in the LUT closest to this signal. Based on the LUT predistorter, an adaptive algorithm was used in a previous study to dynamically learn the weights stored in the LUT by training the normalized least mean squares (NLMS) error between the transmitted signal and the distorted signal as criterion and train with a stochastic-gradient-based approach [12], and thereafter the NLMS-based predistorter was capable of tracking changes in LED characteristics. In another study, by focusing on the fact that the distortion resulting from the LED is nonlinear, a nonlinear transformation of the input was introduced by a Chebyshev polynomial expansion and the weights were also trained by a stochastic-gradient-based approach [13].

Fundamentally, using a predistorter to compensate for the LED nonlinear input power to the output illumination function $\mathcal{I}(p, \Theta)$ consists of cascading a block that exhibits its inverse function $\mathcal{I}^{-1}(v, \boldsymbol{\Theta})$ in front of the LED, as shown in Figure 1. The major issue that particularly needs to be addressed is how to accurately approximate and train the inverse function $\mathcal{I}^{-1}(v, \boldsymbol{\Theta})$ when the LED time-variant nonlinearity function $\mathcal{I}(p, \boldsymbol{\Theta})$ is changed due to temperature variation, aging, or other physical changes denoted by a time-variant state vector $\boldsymbol{\Theta}\left(t_{i}\right)$ at time instant $t_{i}$. That is, the LED nonlinearity should be dynamically learned and compensated for by using its approximated inverse function with an adaptive predistorter. 


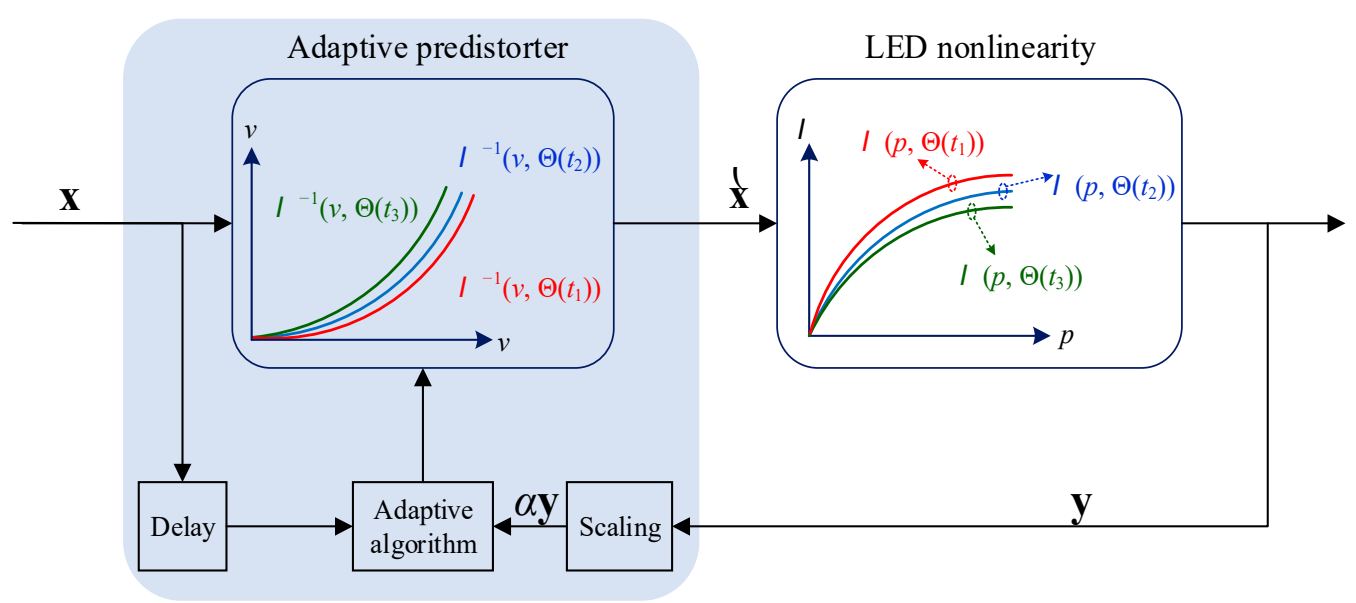

Figure 1. Illustration of the application of an adaptive predistorter to compensate for LED time-variant nonlinearity.

As for function approximation, artificial neural networks (ANNs) are considered to be a powerful tool according to the associated and well-known universal approximation theory [14]. It was the recognition of this power that motivated the consideration of an ANN-based predistortion algorithm in the present study. In addition, an adaptive training method was designed to make the proposed ANN-based predistorter capable of tracking the LED characteristics due to temperature variation and aging. Meanwhile, although the existing works reported in the literature were mainly based on simulations without experimental verifications [11-13], in the present study, laboratory experiments utilizing physical devices were conducted to verify the theoretical analysis of the predistortion algorithm.

The remainder of this paper is organized as follows. Section 2 presents a system model of adaptive predistorters for OFDM-based VLC. The proposed ANN-based predistortion is then presented in Section 3. Section 4 describes the simulation results with experimental verifications. Finally, the conclusions of the study are presented in Section 5.

\section{Preliminary Work}

\subsection{System Model}

It is thought that the computational complexity of DCO-OFDM is lower than that of ACO-OFDM for a fixed symbol rate [15]. However, DCO-OFDM signals would suffer from more serious LED nonlinearity than would ACO-OFDM signals because DCO-OFDM converts real-value bipolar signals to polar ones by adding a DC bias, a distinction which suggested the consideration of adaptive predistortion in the context of DCO-OFDM-based VLC systems in this study. Figure 2 is a block diagram of a DCO-OFDM VLC system with an adaptive predistorter. The input of the DCO-OFDM modulation block is represented as $\mathbf{X}=\left[X_{0}, X_{1}, \cdots, X_{N-1}\right]$, where $X_{k}$ denotes the $M$-ary quadrature amplitude modulated (QAM) complex data signal on the $k$-th subcarrier and $N$ is the size of the IFFT. Moreover, $X_{k}$ in $\mathbf{X}$ should have Hermitian symmetry in order to have a real time-domain signal, and should be followed by adding a DC bias for IM-DD optical wireless systems [6,7]. Using the IFFT, the time-domain signal corresponding to $\mathbf{X}$ is given by $\mathbf{x}=\left[x_{0}, x_{1}, \cdots, x_{N-1}\right]$, where the $n$-th sample is given by:

$$
x_{n}=\frac{1}{N} \sum_{k=0}^{N-1} X_{k} \exp \left(\frac{j 2 \pi n k}{N}\right),
$$

and the bipolar real signal $\mathbf{x}$ is converted to a positive DCO-OFDM signal $\mathbf{x}^{(\mathrm{DCO})}=$ $\left[x_{0}^{(\mathrm{DCO})}, x_{1}^{(\mathrm{DCO})}, \cdots, x_{N-1}^{(\mathrm{DCO})}\right]$ by adding a DC bias $x_{D C}$ to $\mathbf{x}$. The optimal DC bias is related to the number of subcarriers and the modulation order, and for the convenience of using different levels of modulation, a fixed DC bias was adopted in this study. Signal $\mathbf{x}^{(\mathrm{DCO})}$ is then predistorted and digital-to-analog (D/A) converted to a continuous electrical 
waveform $x(t)$. The LED, serving as an optical modulator, transforms $x(t)$ into an optical signal, and the resulting signal is transmitted through the optical channel. Usually, the VLC channel is a line-of-sight (LOS) channel and the dominant shot noise is modeled as additive white Gaussian noise (AWGN) [16]. The received light intensity $r(t)$ is perceived by utilizing a PD in the receiver. The analog signal is analog-to-digital (A/D) converted and down-sampled to be $\hat{\mathbf{x}}^{(\mathrm{DCO})}$ and processed by the demodulation block to attain estimates of $\mathbf{X}$, which is denoted as $\hat{\mathbf{X}}$.

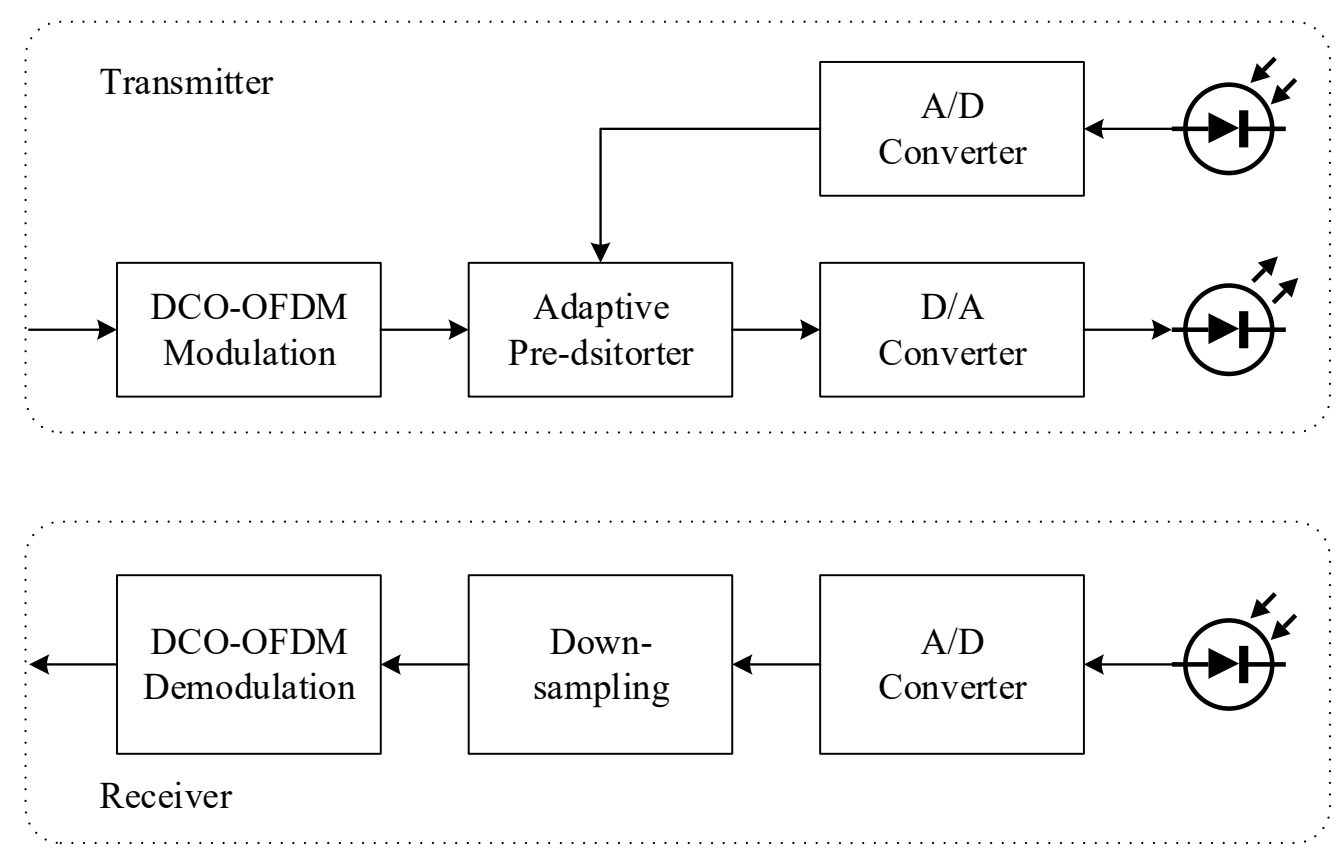

Figure 2. Illustration of DCO-OFDM VLC system with adaptive predistorter.

\subsection{Adaptive Predistortion}

The LED is a nonlinear device, and its behavior can be modeled using the measured input and output characteristics of the adopted LED. To compensate for the LED nonlinearity, a predistortion block is applied to $\mathbf{x}^{(\mathrm{DCO})}$, as shown in Figure 2. In the transmitter, a PD is used to build a feedback signal $y(t)$ to serve as a reference signal for the sake of adaptively adjusting the predistorter to make it capable of tracking the LED characteristics due to temperature variation and aging.

Generally, an error signal $\mathbf{e}^{(\mathrm{DCO})}$, the difference between $\mathbf{x}^{(\mathrm{DCO})}$ and $\alpha \mathbf{y}^{(\mathrm{DCO})}$, where $\alpha$ is a scaling factor, has been used to update parameters in adaptive predistorters, and an NLMS-based algorithm has been proposed to update LUTs [12]. Assuming that at time instant $t$, the $i$-th signal $x_{i}^{(\mathrm{DCO})}$ in $\mathbf{x}^{(\mathrm{DCO})}$ would be in the range of the $k$-th partition cell in the LUT, and it would be predistorted by the representation value of that cell $w_{k}^{(t)}$, then the $k$-th representation value could be updated as follows:

$$
w_{k}^{(t+1)}=w_{k}^{(t)}+\mu_{k} e_{i}^{(\mathrm{DCO})} x_{i}^{(\mathrm{DCO})},
$$

where $e_{i}^{(\mathrm{DCO})}=x_{i}^{(\mathrm{DCO})}-\alpha y^{(\mathrm{DCO})}$ and $\mu_{k}$ denotes the step size for the NLMS algorithm [12]. Meanwhile, Chebyshev polynomials have been used to approximate the inverse of the LED nonlinearity as compared to just using a predistortion factor in the NLMS-based predistorter [13]. In the Chebyshev polynomial-based predistorter, the $k$-th representation value was updated as follows:

$$
w_{k}^{(t+1)}=w_{k}^{(t)}+\mu_{k} e_{i}^{(\mathrm{DCO})} T_{j}\left(x_{i}^{(\mathrm{DCO})}\right),
$$


where $T_{j}$ denotes the $j$-th Chebyshev polynomial of the first kind.

\section{The Proposed ANN-Based Predistorter}

A schematic diagram of the proposed artificial neural network (ANN)-based predistorter, which has the structure of a multilayer perceptron, is shown in Figure 3, in which $N_{I}, N_{H, j}$, and $N_{O}$ denote the neuron in the input layer, the $j$-th neuron in the hidden layer, and the neuron in the output layer, respectively. ANNs were invented to serve as powerful computing systems and were inspired by the constitution of biological brains. As shown in Figure 3a, the proposed ANN-based predistorter constitutes the input layer, one hidden layer, and the output layer. Several neurons, the basic units in an ANN, could be included in each layer. As shown in Figure $3 b$, a specified neuron $j$ would be connected with $K$ inputs $\mathbf{m}=\left[\begin{array}{llll}m_{1} & m_{2} & \cdots & m_{K}\end{array}\right]$ by links with adjustable weights $\mathbf{w}=\left[\begin{array}{llll}w_{1 j} & w_{2 j} & \cdots & w_{K j}\end{array}\right]$, and a bias term $b_{j}$. An activation function $f(\cdot)$ is used to introduce nonlinearity into the output of a neuron when a set of inputs and a bias term are given. Thus, the output of the $j$-th neuron in the hidden layer, $z_{N_{H, j}}$ is represented as follows:

$$
z_{N_{H, j}}=f^{\left(N_{H, j}\right)}\left(w^{\left(N_{H, j}\right)} u+b^{\left(N_{H, j}\right)}\right),
$$

where $u$ is the input to $N_{I}$, and $w^{\left(N_{H, j}\right)}, b^{\left(N_{H, j}\right)}$, and $f^{\left(N_{H, j}\right)}(\cdot)$ represent the weight, the bias value, and the activation function of $N_{H, j}$, respectively. Furthermore, the output of the neuron in the output layer, $z_{N_{O}}$, is represented as follows:

$$
z_{N_{O}}=f^{\left(N_{O}\right)}\left(\sum_{j=1}^{J} w_{j}^{\left(N_{O}\right)} z_{N_{H, j}}+b^{\left(N_{O}\right)}\right),
$$

where $w_{j}^{\left(N_{O}\right)}, b^{\left(N_{O}\right)}$, and $f^{\left(N_{O}\right)}(\cdot)$ are the weight associated with the $j$-th input, the bias value, and the activation function of $N_{O}$, respectively.

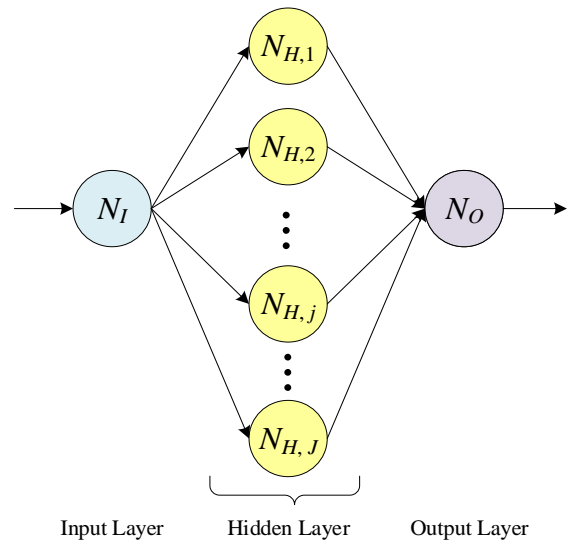

(a)

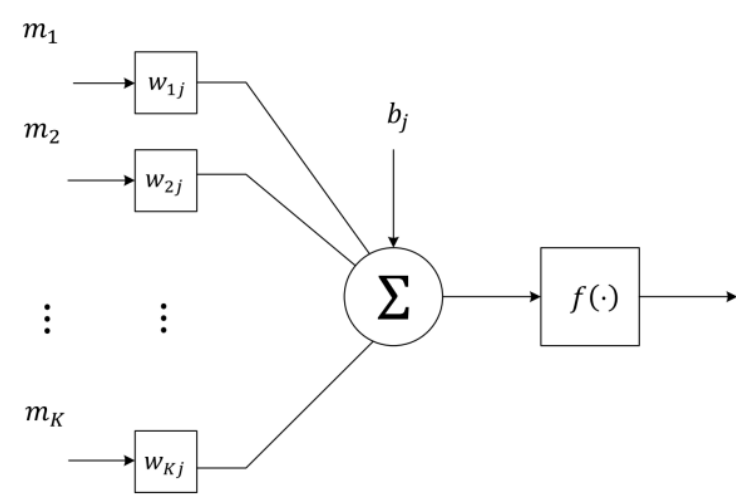

(b)

Figure 3. Illustration of the proposed ANN predistorter: (a) The structure of an artificial neural network; (b) the structure of a specified neuron $j$.

The output of the proposed ANN predistorter, $\mathbf{z}_{N_{O}}$, is a cascade of nonlinear transformation of (4) and (5). When a set of training data $\mathbf{u}$ is applied to this ANN predistorter, its corresponding output $\mathbf{z}_{N_{O}}$ can be mathematically expressed as:

$$
\mathbf{z}_{N_{O}}=F(\mathbf{u}, \boldsymbol{\varphi}),
$$

where $F$ stands for the cascade function of all nonlinear transforms of the neurons in each layer and $\varphi$ denotes the weights and bias values in the neural network. The optimal values 
in $\varphi$ are usually learned on a training set, with known desired outputs. To start the training process, $\varphi$ was initially chosen at random.

A set of input values and expected values matching them should be provided to execute the training process. As shown in Figure 1, the aim of the predistorter was to pre-emphasize the input signal $\mathbf{x}^{(\mathrm{DCO})}$ in order to maintain this pre-emphasized signal as a replica of $\mathbf{x}^{(\mathrm{DCO})}$ after the distortion by the LED nonlinearity. This indicates that the predistortion function is an inverse of the LED nonlinearity, which suggests the use of the captured outputs of the LED, $\mathbf{y}^{(\mathrm{DCO})}$, as the inputs to the predistorter and that its corresponding outputs are $\mathbf{x}^{(\mathrm{DCO})}$. That is:

$$
F\left(\alpha \mathbf{y}^{(\mathrm{DCO})}, \boldsymbol{\varphi}\right)=\mathbf{x}^{(\mathrm{DCO})}
$$

Based on (7), the optimal values in $\varphi$ were trained in the present study by using a Levenberg-Marquardt (LM) backpropagation algorithm. The LM algorithm was derived from Newton's method for the data fitting of a nonlinear function and was developed for the ANN based on the advantage that its training speed is 10 to 100 times faster than that of the usual gradient descent backpropagation method $[17,18]$.

The main contributions of the proposed ANN predistorter in comparison with the methods in previous literatures are summarized as follows. First, the nonlinear ANN was studied to establish function approximation to the inverse function of the nonlinear characteristic curve of LED. Based on universal approximation theorem, ANN can approximate any continuous function and thus it can prevent the predistorter from quantization error as in $[11,12]$. Moreover, based on the relationship between the inputs and the corresponding outputs of the LED device, to adopt a real nonlinear characteristic curve of LED truly represents a more realistic LED nonlinear effect to the DCO-OFDM VLC transmission. Second, in the study, laboratory experiments utilizing physical devices were conducted to verify the theoretical analysis of the predistortion algorithm. In view of most literatures related to LED linearization only presenting their results using computer simulations, the experimental results based on the self-built DCO-OFDM VLC testbed presented in this study would make a constructive contribution for the design of the DCO-OFDM VLC transmission.

\section{Simulations and Measured Results}

The relationship between the dissipated power and the luminous flux is used in the paper to describe the nonlinear behavior of an LED. A phosphor-based white LED (Crescent D01-A1-A33) was measured by using an integrating sphere (ISUZU SLM-12), and its dissipated power versus the output luminous flux is shown in Table 1. To implement a unified performance simulator, a normalized LED transfer function, as plotted in Figure 4, was used in the following performance evaluation of different considered predistortion algorithms. A Rapp-like model was adopted to fitting the normalized LED transfer function as follows [19]:

$$
\Phi_{\text {norm }}\left(p_{d, \text { norm }}\right)=\frac{h\left(p_{d, \text { norm }}\right)}{\left(1+\left(\frac{h\left(p_{d, \text { norm }}\right)}{p_{d, \max }}\right)^{2 k}\right)^{1 / 2 k}}
$$

where $p_{d, \text { max }}$ is the maximum normalized dissipated power through the LED, $h\left(p_{d, n o r m}\right)$ is the function describing the measured characteristics between the normalized dissipated power $p_{d, n o r m}$ and the normalized luminous flux $\Phi_{n o r m}$, and $k$ is the knee factor which controls the smoothness of the transition from the linear region to the saturation region. As shown in Figure 4, the Rapp-like model with knee factor $k=3.0$ had a better fitting to the measured data of the considered LED and was adopted in the performance simulations of the LED nonlinearity predistorter detailed below. 
Table 1. The measurements of the LED (Crescent D01-A1-A33).

\begin{tabular}{cc}
\hline Dissipated Power $\boldsymbol{P}_{\boldsymbol{d}}(\mathbf{m W})$ & Luminous Flux $\boldsymbol{\Phi}(\mathbf{l m})$ \\
\hline 0.83 & 0 \\
19.77 & 0 \\
127.6 & 7474 \\
291.5 & 17,314 \\
497.4 & 19,434 \\
\hline
\end{tabular}

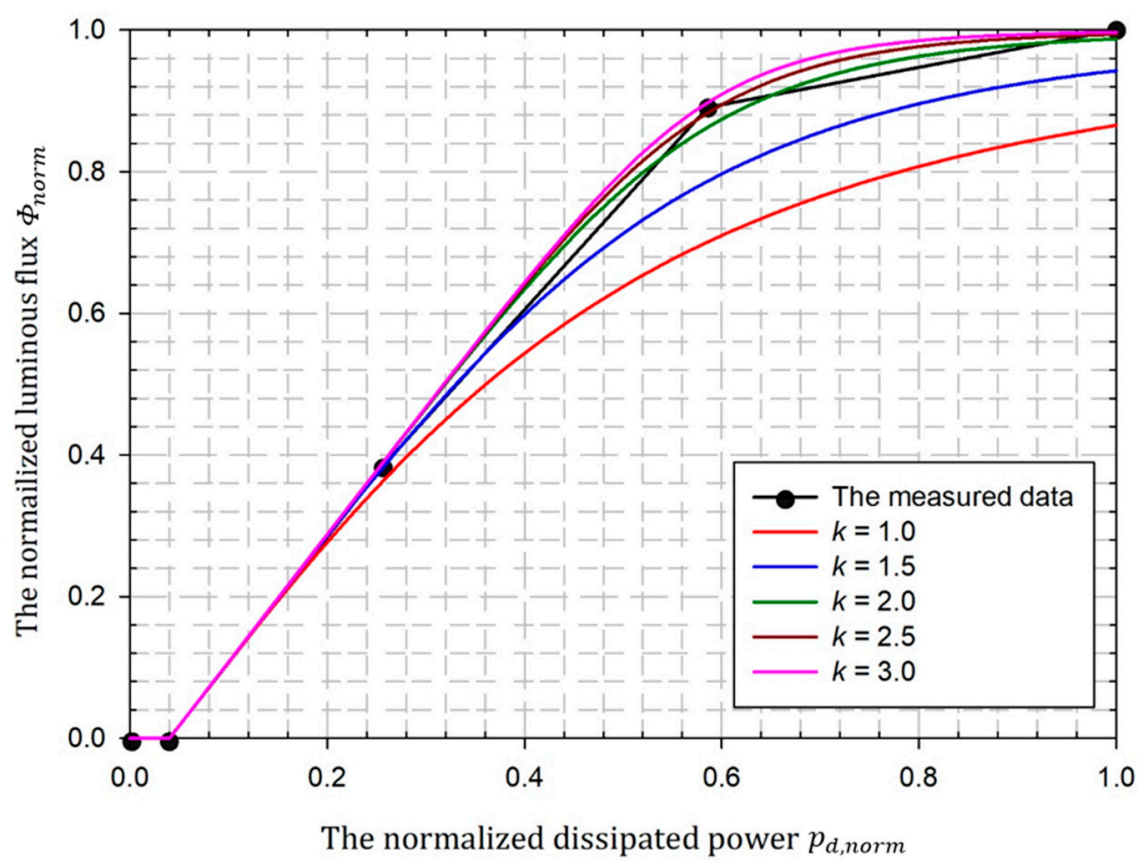

Figure 4. The characteristic function of the adopted LED.

In the conducted computer simulations, $10^{5}$ independent DCO-OFDM symbols were randomly generated with the parameters summarized in Table 2 . To find the proper settings of the number of ANN training symbols $N_{\text {train }}$ and the number of neurons in the hidden layer of the ANN predistorter $J$, the BER versus the combination of the number of ANN training symbols and the number of hidden nodes are shown in Figure 5. Generally, as can be seen in Figure 5, the BER of the DCO-OFDM with the proposed ANN predistorter was decreased as the number of ANN training symbols was increased. The received scaling DCO-OFDM symbol $\alpha \mathbf{y}^{(\mathrm{DCO})}$ was used to train the proposed ANN predistorter. When the setting of $N_{\text {train }}=1$, it showed a limitation in effectively training the parameters in $\varphi$ because the distribution of the time-domain samples in one DCO-OFDM symbol might have been quite non-uniform in the domain of the desired predistorter function. When the value of $N_{\text {train }}$ was increased, more time-domain samples in the $N_{\text {train }}$ DCOOFDM symbols were available, and these samples might have had a wider and uniform distribution within the domain of the desired predistorter function. As can be seen in Figure 5, the adaptation of $N_{\text {train }}=5$ seems to have been enough to effectively train the parameters in $\varphi$ of the predistorter function. Moreover, the simulation results presented in Figure 5 indicated that the proposed ANN predistorter composed of a single hidden layer with 4 neurons was able to achieve a stable BER performance. Meanwhile, increasing the number of neurons in the hidden layer to more than 4 only seemed to increase the complexity of the network; thus, the number of neurons in the hidden layer $J$ was set at 4 . 
Table 2. Summary of simulation parameters.

\begin{tabular}{cc}
\hline Parameters & Value \\
\hline Subcarriers number, $N$ & 128 \\
QAM Modulation order, $\boldsymbol{M}$ & $\{16,64\}$ \\
DC bias in DCO-OFDM, $\boldsymbol{x}_{\boldsymbol{D} C}(\mathrm{~dB})$ & 13 \\
Number of ANN training symbols, $\boldsymbol{N}_{\text {train }}$ & $\{1,2,3,4,5,6\}$ \\
Number of neurons in the hidden layer, $\boldsymbol{J}$ & $\{3,4,5,6\}$ \\
Number of hidden layers, $\boldsymbol{N}_{\boldsymbol{h}}$ & $\{1,2,3\}$ \\
Knee factor in Rapp model, $\boldsymbol{k}$ & 3.0 \\
\hline
\end{tabular}

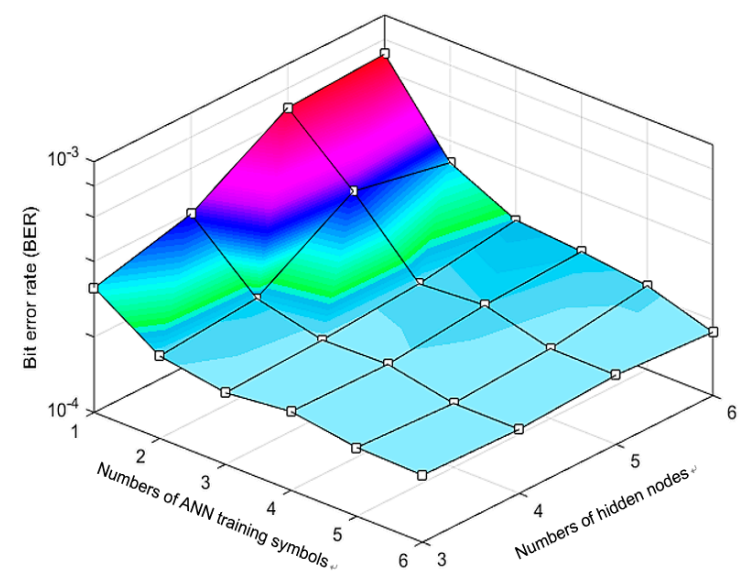

(a)

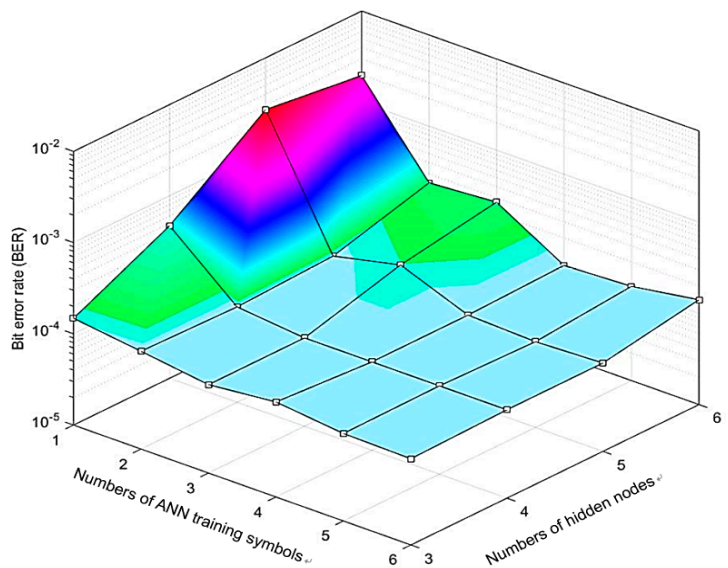

(b)

Figure 5. Illustration of the proposed ANN predistorter: (a) 16 QAM DCO-OFDM with $E_{b} / N_{0}=25 \mathrm{~dB}$; (b) 64 QAM DCO-OFDM with $E_{b} / N_{0}=30 \mathrm{~dB}$.

The convergence of the proposed ANN predistorter with different numbers of neurons in the hidden layer is shown in Figure 6. Consistent with the findings of Figure 5, the ANN with a 4-neuron hidden layer achieved the minimum mean-square error. The impacts of increasing the number of hidden layers are shown in Figures 7 and 8. Intuitively, the adoption of more layers in an ANN seemed to result in better achievable BER. However, the proposed ANN predistorter did not benefit from an increase in the number of hidden layers. This was mainly because the feedback replica of the original electrical source through PD, used as training set for the ANN, was noisy. The ANN became increasingly complicated with each increase in the number of hidden layers, and thus it could not be well trained using this noisy training set. In addition, the inverse function to the characteristic function of the adopted LED is expected to be simple and monotonic. As such, using more hidden layers might cause the ANN to be particularly sensitive to the noise, rather than the desired signal, resulting in overfitting.

The BERs of several LED predistortion algorithms, including the NLMS [12], the Chebyshev polynomial [13], and the proposed ANN predistortion, plotted against the electrical energy per bit to single-sided noise power spectral density $E_{b} / N_{0}$ for the cases of 16 QAM $(M=16)$ and 64 QAM $(M=64)$ are shown in Figures 9 and 10, respectively. In the cases of both 16 QAM and 64 QAM, it was evident that the BERs reached by the proposed ANN predistortion were lower than those reached by using the NLMS and the Chebyshev polynomial. The NLMS had the worst performance since the NLMS only used a one-dimensional LUT to approximate the predistorter function. In contrast, the Chebyshev polynomial method achieved a better performance than the NLMS method because the Chebyshev polynomial method adds polynomial expansion compensation terms onto the predistortion factors stored in the LUT. Meanwhile, the proposed ANN method, using a cascade of nonlinear functions to approximate the nonlinear predistorter 
function, was capable of revealing the LED nonlinearity and could adaptively learn it through the training set, i.e., through the scaling feedback signal $\alpha \mathbf{y}^{(\mathrm{DCO})}$.

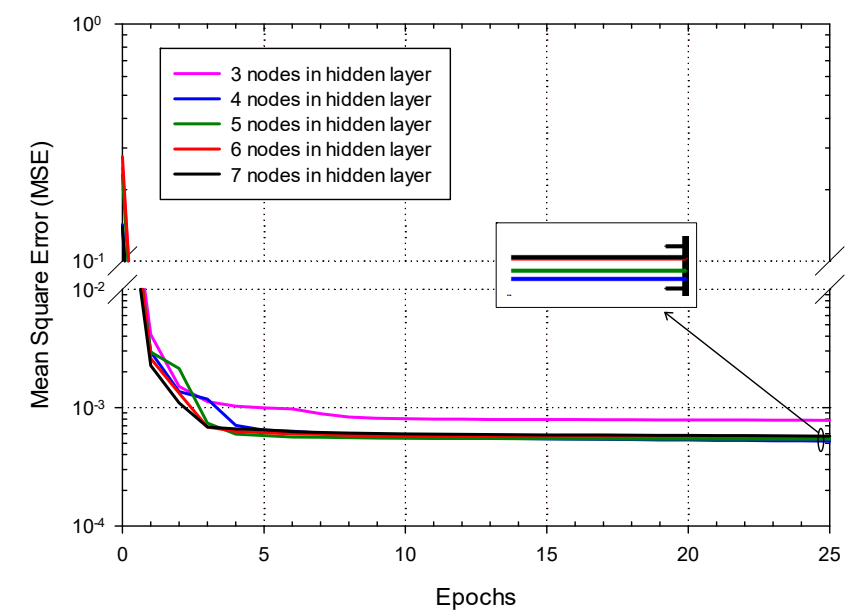

Figure 6. Convergence of the proposed ANN predistorter with different nodes in the hidden layer.

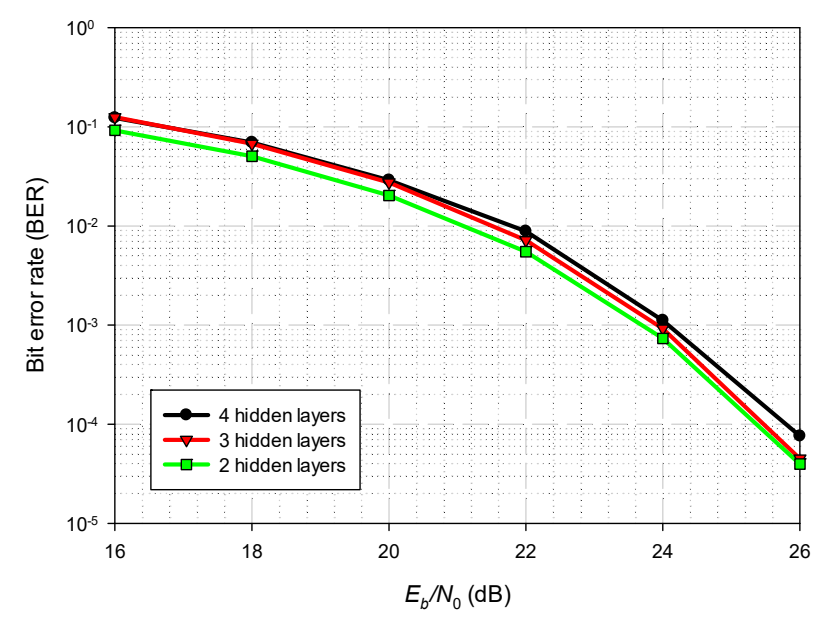

Figure 7. BER in AWGN versus $E_{b} / N_{0}$ of the proposed ANN predistorter for different numbers of hidden layers with 16 QAM.

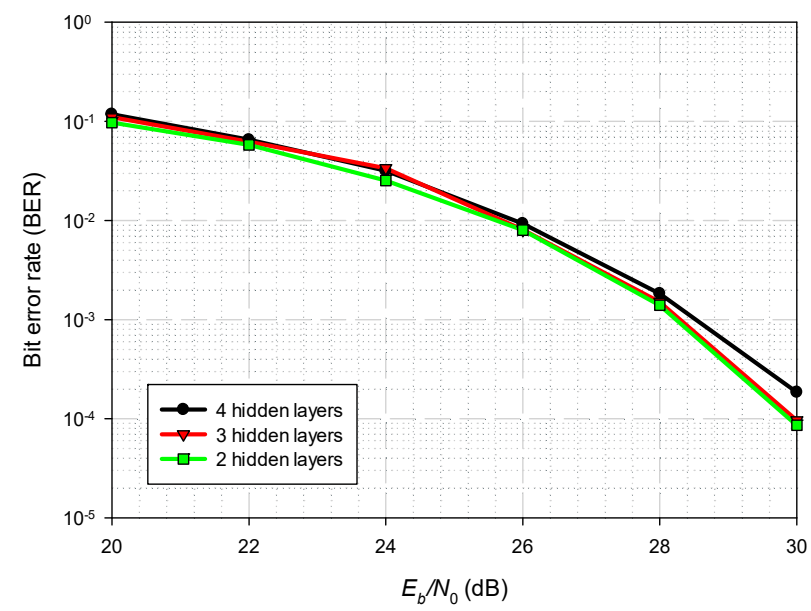

Figure 8. BER in AWGN versus $E_{b} / N_{0}$ of the proposed ANN predistorter for different numbers of hidden layers with 64 QAM. 


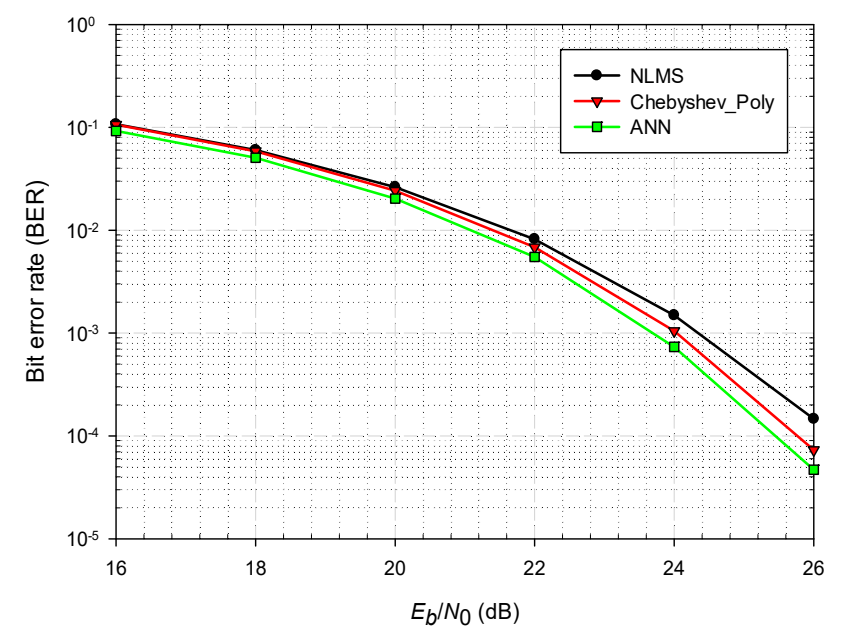

Figure 9. BER in AWGN versus $E_{b} / N_{0}$ for the NLMS, the Chebyshev polynomial, and the proposed ANN predistortion with 16 QAM.

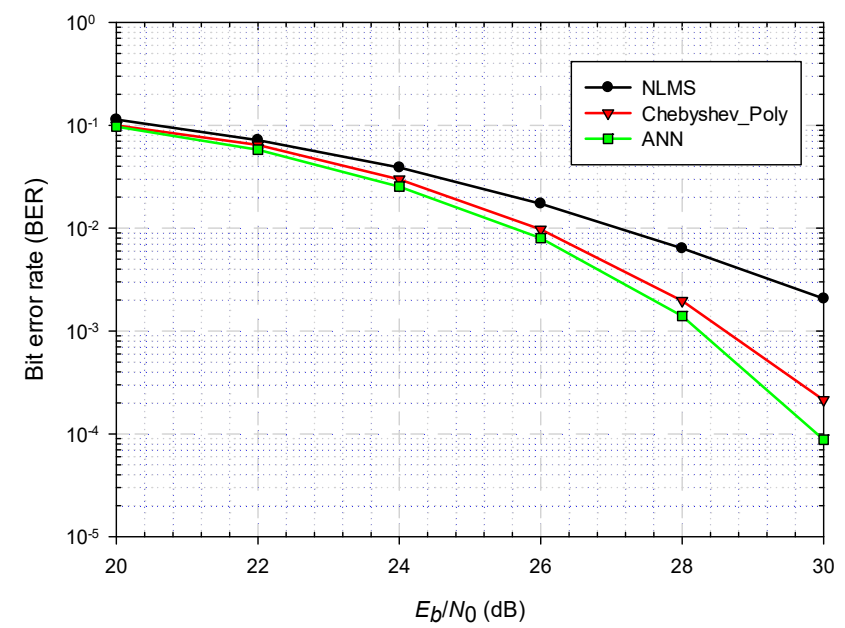

Figure 10. BER in AWGN versus $E_{b} / N_{0}$ for the NLMS, the Chebyshev polynomial, and the proposed ANN predistortion with 64 QAM.

Notably, there were noise components contained in $\alpha \mathbf{y}^{(\mathrm{DCO})}$, and this resulted in almost no performance difference among the NLMS, the Chebyshev polynomial, and the proposed ANN predistortion when the system was under a low $E_{b} / N_{0}$ scenario. In contrast, in the scenario of moderate $E_{b} / N_{0}$, the quality of $\alpha \mathbf{y}^{(\mathrm{DCO})}$ was better, and thus the proposed ANN predistortion was apparently able to manifest its superiority over the NLMS and Chebyshev polynomial methods in that scenario.

The effectiveness of the ANN predistorter in a DCO-OFDM VLC system was also validated in this study through experimental verification. The system setup of the selfbuilt DCO-OFDM VLC testbed is shown in Figure 11. A pseudo-random binary sequence was programmed, modulated, and predistorted using the considered predistortion algorithms in the computer. To conduct the D/A conversion, the computer was connected to an arbitrary waveform generator (AWG) (Tektronix AFG3151C) under the constraint of modulation bandwidth of the LED. Through the bias tee (Tektronix PSPL5575A), the generated waveform signal was superimposed onto the bias current to drive the adopted LED (Crescent D01-A1-A33). On the basis of the measured data of the LED in Table 1, the bias current was set to $60 \mathrm{~mA}$ and the mean of the generated waveform signal was set to 5 volts, and these settings caused the operation point of the LED to be $300 \mathrm{~mW}$ in order to introduce the nonlinearity effect into the LED emitting optical signal. 


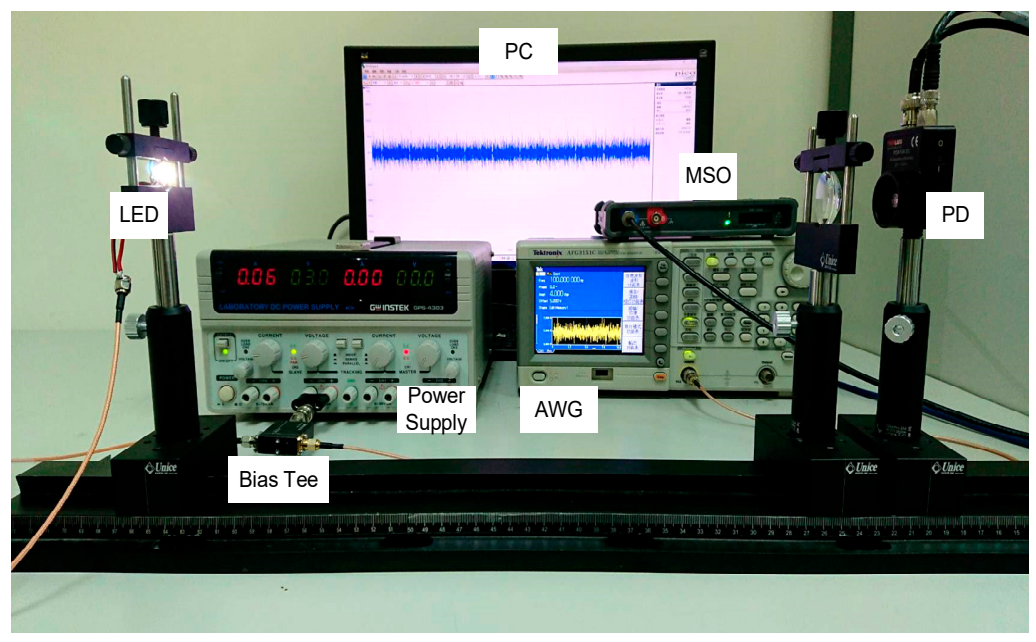

Figure 11. Experimental setup of the DCO-OFDM VLC testbed.

A PD (THORLABS PDA10A-EC), with a $150 \mathrm{MHz}$ bandwidth, $1 \mathrm{~mm}^{2}$ active area, and $5 \times 10^{-3} \mathrm{~V} / \mathrm{A}$ transimpedance gain, was utilized to direct detect the light intensity. To conduct the A/D conversion, the PD output was captured by using a mixed signal oscilloscope (MSO) (PicoScope 3205D), and then followed by a process of downsampling. Finally, the sampled signal was further subjected to packet synchronization, demodulation, and BER analysis. Figure 12 shows constellation diagrams of 16 QAM and 64 QAM modulation under the condition that the distance between the LED and the PD was $40 \mathrm{~cm}$. Obviously, the constellation diagrams with the ANN predistorter are much clearer than those with either the NLMS or Chebyshev polynomial predistorters. This observation was also validated by the error vector magnitude (EVM) results, which are shown in the titles of each subfigure.

Moreover, the BERs of the considered LED predistortion algorithms plotted against the distances between the LED and the PD for the cases of 16 QAM $(M=16)$ and 64 QAM $(M=64)$ are shown in Figures 13 and 14, respectively. As can be seen in Figure 13, the experimental results verified that the BER of the 16 QAM modulated OFDM system with the proposed ANN predistorter was significantly superior to those with the NLMS and the Chebyshev polynomial predistorters. As for the case of the 64 QAM-modulated OFDM signals shown in Figure 14, the BER of the system with the proposed ANN predistorter still outperformed those with the NLMS and the Chebyshev polynomial predistorters. In summary, the experimental results validated the view that the proposed ANN predistorter was capable of adequately compensating for LED nonlinearity, and hence achieving better system performance compared to the NLMS and the Chebyshev polynomial predistorters. 
$\mathrm{EVM}=-18.03 \mathrm{~dB}$

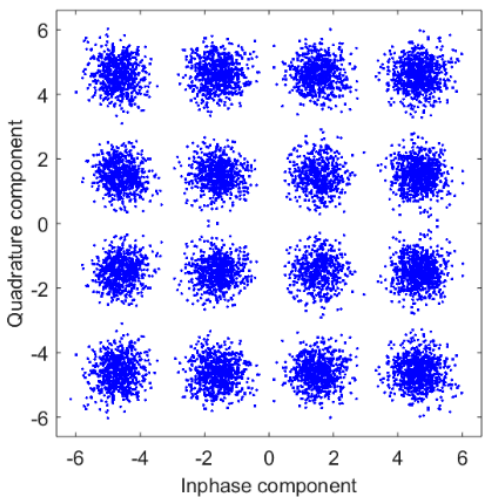

(a)

$\mathrm{EVM}=-16.35 \mathrm{~dB}$

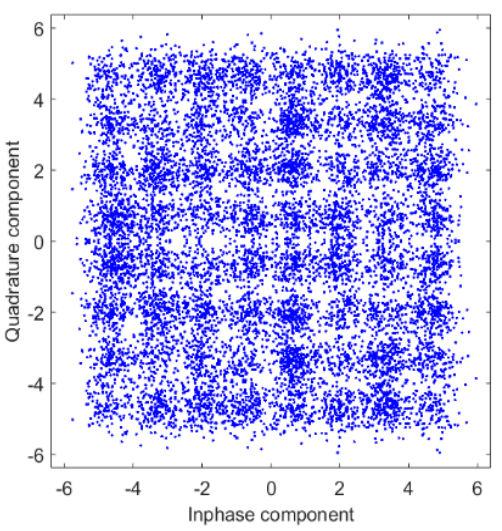

(d)
$\mathrm{EVM}=-19.70 \mathrm{~dB}$

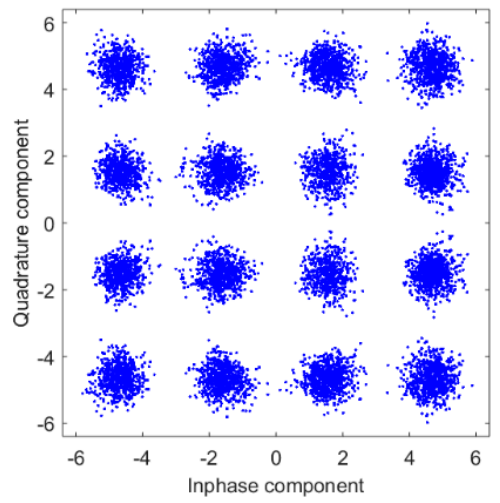

(b)

$\mathrm{EVM}=-18.82 \mathrm{~dB}$

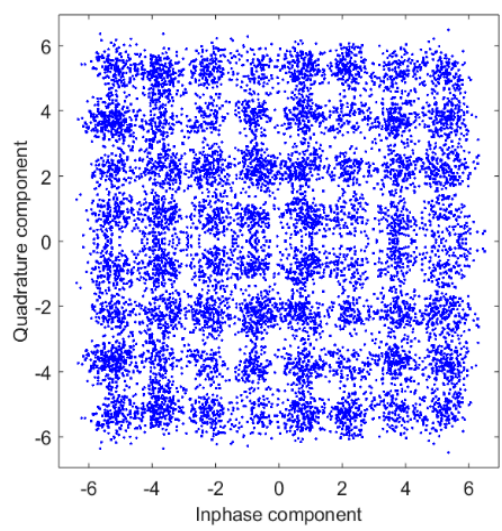

(e)
$\mathrm{EVM}=-24.95 \mathrm{~dB}$

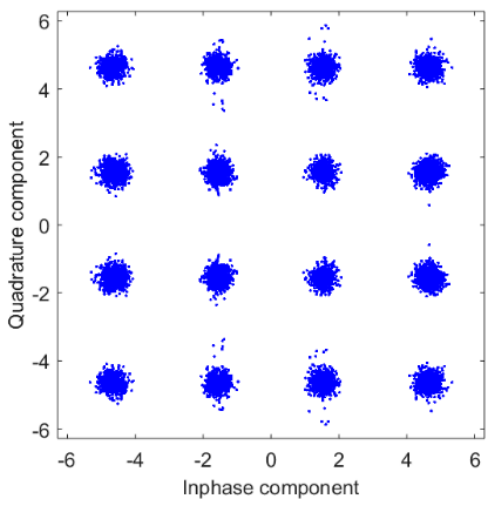

(c)

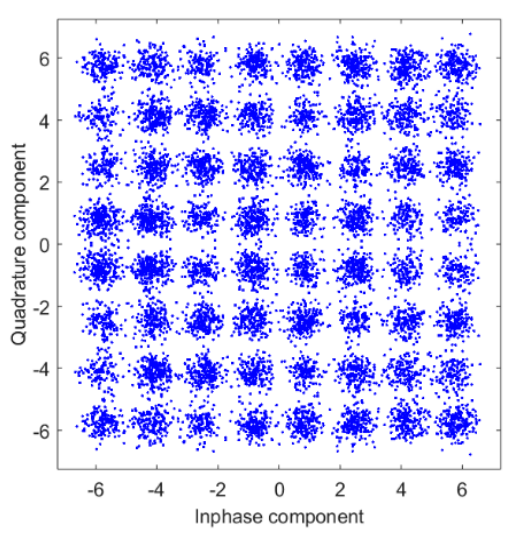

(f)

Figure 12. Constellation diagrams of 16 QAM and 64 QAM modulation. (a-c) are constellation diagrams of the 16 QAM modulated OFDM signal with the NLMS, Chebyshev polynomial, and the proposed ANN predistorters, respectively. (d-f) are constellation diagrams of the 64 QAM modulated OFDM signal with the NLMS, Chebyshev polynomial, and the proposed ANN predistorters, respectively.

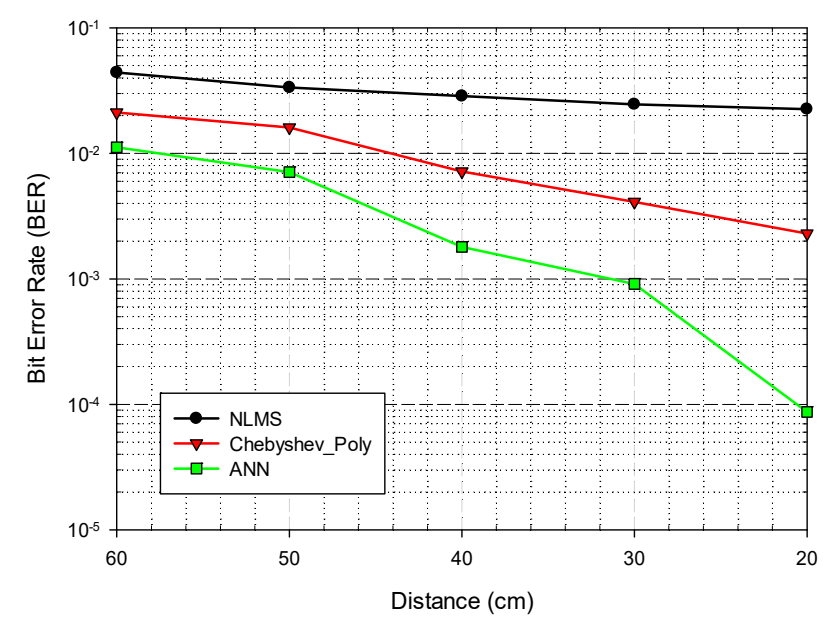

Figure 13. BER versus the distance between the LED and the PD of the 16 QAM modulated OFDM for the NLMS, the Chebyshev polynomial, and the proposed ANN predistortion. 


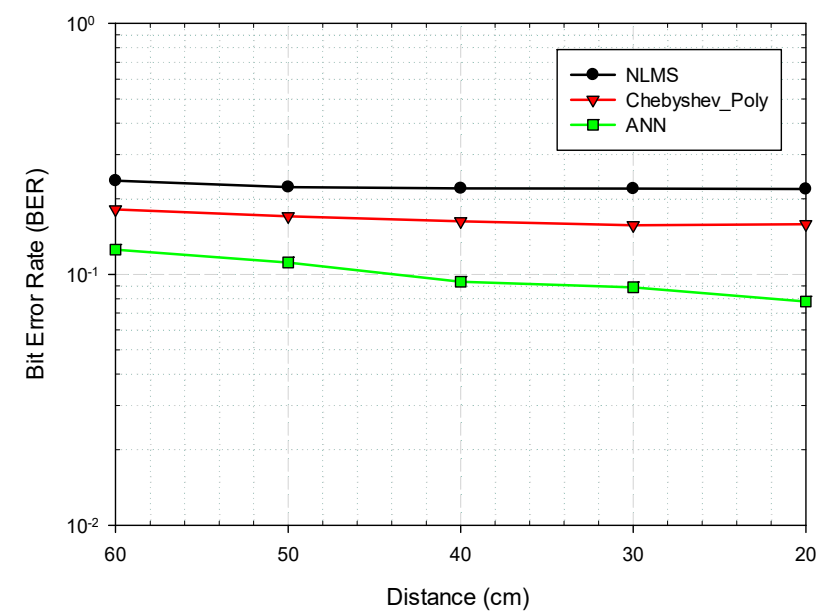

Figure 14. BER versus the distance between the LED and the PD of the 64 QAM modulated OFDM for the NLMS, the Chebyshev polynomial, and the proposed ANN predistortion.

\section{Conclusions}

Nonlinearity inherited in the LED severely degrades the performance of OFDM VLC systems due to the high PAPR in OFDM signals. In this paper, we proposed the use of an ANN predistorter to pre-emphasize the DCO-OFDM VLC signal. The nonlinearity effects of the LED could be adequately compensated for with the capability of universal function approximation of the ANN. As observed from both computer simulations and experimental verification, the ANN predistorter achieved considerable performance improvement over the existing NLMS and Chebyshev polynomial predistortion methods in terms of the BER and EVM of the received signal constellation.

Author Contributions: Conceptualization, J.-K.L.; methodology, J.-K.L.; software, J.-K.L. and Y.-H.C.; validation, J.-K.L. and Y.-H.C.; formal analysis, J.-K.L. and Y.-H.C.; investigation, J.-K.L. and Y.-H.C.; resources, J.-K.L.; data curation, J.-K.L. and Y.-H.C.; writing-original draft preparation, J.-K.L.; writing-review and editing, J.-K.L.; visualization, J.-K.L. and Y.-H.C.; supervision, J.-K.L.; project administration, J.-K.L.; funding acquisition, J.-K.L. All authors have read and agreed to the published version of the manuscript.

Funding: This research received no external funding.

Data Availability Statement: The data presented in this study are all available in this article.

Conflicts of Interest: The authors declare no conflict of interest.

\section{References}

1. Uysal, M.; Miramirkhani, F.; Narmanlioglu, O.; Baykas, T.; Panayirci, E. IEEE 802.15.7r1 Reference Channel Models for Visible Light Communications. IEEE Commun. Mag. 2017, 55, 212-217. [CrossRef]

2. Ma, H.; Lampe, L.; Hranilovic, S. Hybrid Visible Light and Power Line Communication for Indoor Multiuser Downlink. IEEE/OSA J. Opt. Commun. Netw. 2017, 9, 635-647. [CrossRef]

3. Burton, A.; Bentley, E.; Minh, H.-L.; Ghassemlooy, Z.; Aslam, N.; Liaw, S.-K. Experimental Demonstration of A 10BASE-T Ethernet Visible Light Communications System using White Phosphor Light-Emitting Diodes. IET Circuits Devices Syst. 2014, 8, 322-330. [CrossRef]

4. Wu, F.M.; Lin, C.T.; Wei, C.C.; Chen, C.W.; Chen, Z.Y.; Huang, H.T.; Chi, S. Performance Comparison of OFDM Signal and CAP Signal over High Capacity RGB-LED-Based WDM Visible Light Communication. IEEE Photonics J. 2013, 5, 7901507. [CrossRef]

5. Carruthers, J.B.; Kahn, J.M. Multiple-Subcarrier Modulation for Nondirected Wireless Infrared Communication. IEEE J. Select. Areas Commun. 1996, 14, 538-546. [CrossRef]

6. Gonzalez, O.; Perez-Jimenez, R.; Rodriguez, S.; Rabadan, J.; Ayala, A. Adaptive OFDM System for Communications over the Indoor Wireless Optical Channel. IET Proc. Optoelectron. 2006, 153, 139-144. [CrossRef]

7. Armstrong, J.; Lowery, A.J. Power Efficient Optical OFDM. Electron. Lett. 2006, 42, 370-372. [CrossRef]

8. Inan, B.; Jeffrey Lee, S.C.; Randel, S.; Neokosmidis, I.; Koonen, A.M.J.; Walewski, J.W. Impact of LED Nonlinearity on Discrete Multitone Modulation. IEEE/OSA J. Optical Commun. Netw. 2009, 1, 439-451. [CrossRef]

9. Rahmatallah, Y.; Mohan, S. Peak-to-Average Power Ratio Reduction in OFDM Systems: A Survey and Taxonomy. IEEE Commun. Surv. Tut. 2013, 15, 1567-1592. [CrossRef] 
10. Zhang, H.; Yang, L.; Hanzo, L. Piecewise Companding Transform Assisted Optical-OFDM Systems for Indoor Visible Light Communications. IEEE Access. 2017, 5, 295-311. [CrossRef]

11. Elgala, H.; Mesleh, R.; Haas, H. Predistortion in Optical Wireless Transmission Using OFDM. In Proceedings of the 2009 Ninth International Conference on Hybrid Intelligent Systems, Shenyang, China, 12-14 August 2009; pp. 184-189.

12. Kim, J.K.; Hyun, K.; Park, S.K. Adaptive Predistorter using NLMS Algorithm for Nonlinear Compensation in Visible-Light Communication System. Electron. Letters. 2014, 50, 1457-1459. [CrossRef]

13. Mitra, R.; Bhatia, V. Chebyshev Polynomial-Based Adaptive Predistorter for Nonlinear LED Compensation in VLC. IEEE Photonics Technol. Lett. 2016, 28, 1053-1056. [CrossRef]

14. Hornik, K. Approximation Capabilities of Multilayer Feedforward Networks. Neural Netw. 1991, 4, 251-257. [CrossRef]

15. Barros, D.J.F.; Wilson, S.K.; Kahn, J.M. Comparison of Orthogonal Frequency-Division Multiplexing and Pulse-Amplitude Modulation in Indoor Optical Wireless Links. IEEE Trans. Commun. 2012, 60, 153-163. [CrossRef]

16. Kahn, J.M.; Barry, J.R. Wireless Infrared Communications. Proc. IEEE. 1997, 85, 265-298. [CrossRef]

17. Hagan, M.T.; Menhaj, M. Training Feed-Forward Networks with the Marquardt Algorithm. IEEE Trans. Neural Netw. 1994, 5, 989-993. [CrossRef]

18. Hagan, M.T.; Demuth, H.B.; Beale, M.H. Neural Network Design; PWS Publishing: Boston, MA, USA, 1996.

19. Elgala, H.; Mesleh, R.; Haas, H. An LED Model for Intensity-Modulated Optical Communication Systems. IEEE Photonics Technol. Lett. 2010, 22, 835-837. [CrossRef] 